\title{
Smooth Muscle Autoantibodies in Infectious Mononucleosis
}

\author{
E. J. HOLBOROW, E. H. HEMSTED, S. V. MEAD
}

British Medical Fournal, 1973, 3, 323-325

\section{Summary}

Smooth muscle antibodies (S.M.A.) were found in the sera of $81 \%$ of 126 patients over 10 years old with seropositive infectious mononucleosis tested within one month of onset. In 27 patients presenting clinically with infectious mononucleosis but having negative Paul-Bunnell tests the incidence of S.M.A. was $44 \%$. In children 10 years of age or less in these two categories S.M.A. were present in $75 \%$ and $25 \%$ respectively, while in children of similar age not suspected of having infectious mononucleosis the incidence of S.M.A. was $10 \%$. Among 45 adults with past histories of seropositive infectious mononucleosis more than one year before the incidence of S.M.A. was $33 \%$, in contrast to $14 \%$ in 98 subjects with a negative history for infectious mononucleosis.

\section{Introduction}

Smooth muscle autoantibodies (S.M.A.) were first reported in the sera of patients with chronic active hepatitis (Johnson et al., 1965) and later assigned to a group of persistent, chiefly IgG, antibody markers of the autoimmune forms of chronic hepatitis (Doniach and Walker, 1969). S.M.A. have since proved to be more widely distributed, occurring early (predominantly at IgM) in acute viral hepatitis (Farrow et al., 1970) and, in the one study reported so far, in a majority of cases of malignant disease (Whitehouse and Holborow, 1971). We have also found S.M.A. in the sera of some subjects apparently healthy at the time of testing and in certain patients with diseases other than those mentioned.

Recently we have noted S.M.A. in sera from patients with infectious mononucleosis (Holborow, $1972 \mathrm{a}, \mathrm{b}$ ). This paper reports a study of the occurrence of S.M.A. during the acute illness of infectious mononucleosis, during follow-up after recovery, and in subjects with and without a past history of clinical infectious mononucleosis.

\section{Materials and Methods}

S.M.A. are at present detectable only by direct immunofluorescence or immunoperoxidase methods. The substrate is usually cryostat sections of unfixed snap-frozen human or animal tissues containing smooth muscle. We used cryostat sections taken from composite blocks of fresh snap-frozen rat stomach, kidney, and liver. These tissues were chosen because sera which are positive for S.M.A. as shown by immunofluorescent staining of mooth muscle fibres may also give outline staining of liver cells ("polygonal" pattern) or diffuse staining of renal glomeruli or both. Sera were tested \footnotetext{
M.R.C. Rheumatism Unit, Canadian Red Cross Memorial Hospital,
Taplow, Berks

E. J. HOLBOROW, M.D., M.R.C.PATH., Member of Scientific Staff

Department of Pathology, Royal Berkshire Hospital, Reading RG1 5AN

E. H. HEMSTED, M.B., F.R.C.PATH., Consultant Pathologist

S. V. MEAD, F.I.M.L.T., Senior Chief Technician in Haematology
}

at 1/10 dilution as previously described (Farnow et al., 1970). Fluorescein isothiocyanate (FITC) conjugates of Wellcome sheep antihuman immunoglobulin, anti-IgG, and antiIgM were used at dilutions (usually $1 / 32$ ) which gave minimal nonspecific staining by indirect immunofluorescence with normal human sera.

Sections were examined with a Reichert Zetopan microscope fitted with an iodine quartz lamp, Balzer FITC-3 interference filter with cut-off to $0.3 \%$ transmission at $500 \mu \mathrm{m}$ and a Wratten 2B gelatin barrier filter, a dry $40 \times$ objective (NA 0.9 ), and $6 \times$ eye-pieces. The intensity of staining in three areas-smooth muscle fibres in the gastric mucosa, muscularis mucosae, and renal arteriolar walls-was scored for each area as 0 (negative), 1 (doubtful), 2 (definite), or 3 (strong). Sera scoring 8-9 were classed as "strong," 5-7 as "moderate + ," and less than 5 as negative.

\section{Patients}

The following gnoups were studied. The sex distribution was approximately equal; the age distributions are shown in table I. Children aged 10 years or less are considered separately where appropriate, since they have a considerably lower incidence of antibody to E.B. virus than older subjects and thus provide a population in which it should be apparent whether the presence of E.B. antibody correlates with that of S.M.A.

TABLE I-Age Distribution of Patients

\begin{tabular}{|c|c|c|c|c|}
\hline Age & $\begin{array}{c}\text { Group } 1 \text { a } \\
\text { I.M./P.B. + }\end{array}$ & $\begin{array}{l}\text { Group 2 a } \\
\text { "I.M."/P.B.- }\end{array}$ & $\begin{array}{l}\text { Group } 4 \\
\text { I.M. History } \\
(>1 \text { yr })\end{array}$ & $\begin{array}{l}\text { Group } 5 \\
\text { No I.M. } \\
\text { History }\end{array}$ \\
\hline $\begin{array}{l}11-15 \\
16-20 \\
21-25 \\
26-30 \\
31-35 \\
36-40 \\
41-45 \\
46-50 \\
\geqslant 51 \\
\text { Not known }\end{array}$ & $\begin{array}{r}21 \\
53 \\
22 \\
11 \\
6 \\
2 \\
0 \\
1 \\
4 \\
6\end{array}$ & $\begin{array}{l}2 \\
6 \\
3 \\
2 \\
4 \\
3 \\
1 \\
1 \\
1\end{array}$ & $\begin{array}{r}1 \\
10 \\
14 \\
7 \\
3 \\
2 \\
1 \\
\\
\\
7\end{array}$ & $\begin{array}{r}0 \\
30 \\
45 \\
14 \\
1\end{array}$ \\
\hline Total & 126 & 27 & 45 & 98 \\
\hline
\end{tabular}

I.M./P.B. $+=$ Infectious mononucleosis, Paul-Bunnell positive.

Group 1 a, b. Clinical Infectious Mononucleosis, PaulBunnell Positive.-(a) Sera were obtained within one month of onset of symptoms from 126 patients over 10 years of age. The Paul-Bunnell test was positive in all of them (range $1 / 10-1 / 1,280$ ) and the atypical mononuclear cells characteristic of infectious mononucleosis were present in blood films made at the time of testing. Twenty-eight of these patients were followed up and a second specimen of serum was obtained between 8 and 32 weeks after onset. (b) As for group 1 a but containing 12 children 10 years old or less.

Group $2 a, b$. Clinical "Infectious Mononucleosis," PaulBunnell Negative.-(a) A group of 27 patients over 10 years old presenting with clinical features resembling infectious mononucleosis but with consistently negative Paul-Bunnell tests. Some of these patients had atypical mononuclear cells. Sera were taken within one month of onset of symptoms. (b) 
As for group 2 a but containing 47 children aged 10 years or less.

Group 3. Control Children.-A group of 106 children aged 10 years or less seen in the ear, nose, and throat clinic. Infectious mononucleosis was not suspected in these patients and the Paul-Bunnell test was not done.

Group 4. Adults with a History of Infectious Mononucleosis.-This was a group of 45 subjects with laboratory records of having had infectious mononucleosis with a positive Paul-Bunnell test more than one year previously and from whom a current sample of blood was available for the present study; 16 had had infectious mononucleosis less than two years before testing, 10 three to four years before, and 19 mone than four years before.

Group 5.-A group of 98 subjects (94 outpatients, 4 laboratory staff) who gave a history negative for infectious mononucleosis on direct questioning. The age range was 16-30 years.

Group 6.-Cord blood samples were obtained from 66 deliveries taking place in the obstetric department of the Royal Berkshire Hospital during a period of two weeks.

\section{Results}

Group 1 a.-In this group of 126 infectious mononucleosis patients examined within one month on onset $102(81 \%)$ had S.M.A. on testing their sera with anti-immunoglobulin conjugate. The antibody was predominantly IgM. Twenty positive sera were titrated further, and their titres ranged from $1 / 10$ to $1 / 160$. With anti-IgG conjugate staining of smooth muscle was usually absent or doubtful, and only 16 of the acute sera positive with anti-immunoglobulin gave definite taining with anti-IgG as well as with anti-IgM. The smooth muscle staining was graded as strong in 63 and moderate in 39 . When the staining was strong glomerular staining of the kidney was often present and sometimes polygonal staining of the liver also. The incidence of glomerular staining and especially of polygonal staining was difficult to assess accurately, as both varied from definite to doubtful, but about $50 \%$ of the sera strongly positive for S.M.A. gave some degree of diffuse glomerular staining and about $30 \%$ gave recognizable polygonal staining with the anti-immunoglobulin conjugate.

The mean Paul-Bunnell titre of the 102 sera positive for S.M.A. was $1 / 80$, and the mean for the 24 S.M.A.-negative sera was $1 / 20$.

Group 1 b. - Of the 12 children in this group $9(75 \%)$ had IgM S.M.A. (7 strong, 2 moderate).

Group 2.-Among the 27 patients over 10 years old (group 2 a) $12(44 \%)$ had S.M.A. (5 strong, 7 moderate). In two of these staining was seen only with anti-IgG conjugate, in nine only with anti-IgM, and in one with both. Among the 47 children 10 years old or less (group 2 b) 12 were S.M.A. positive (6 strong, 6 moderate); these were all positive with anti-IgM only.

Group 3.-Sera from 106 children were tested with antiimmunoglobulin conjugate only. Eleven were S.M.A. positive (three strong, eight moderate).

Group 4.- Of the 45 healthy individuals in this group 15 (33\%) had S.M.A. (7 strong, 8 moderate). Ten of these were positive only with anti-IgG, four only with anti-IgM, and one with both. There was no correlation of positivity with interval since the initial attack.

Group 5.-Ninety-eight subjects were tested of whom 14 (14\%) had S.M.A. (5 strong, 9 moderate). Four of these positives were IgG and $10 \mathrm{IgM}$.

Group 6.- Of the 66 cond blood sera eight were positive for S.M.A.; all eight were positive only with anti-IgG conjugate.

The results in these six groups are summarized in table II.
TABLE II-Incidence of S.M.A. in Infectious Mononucleosis Patients and Other Subjects

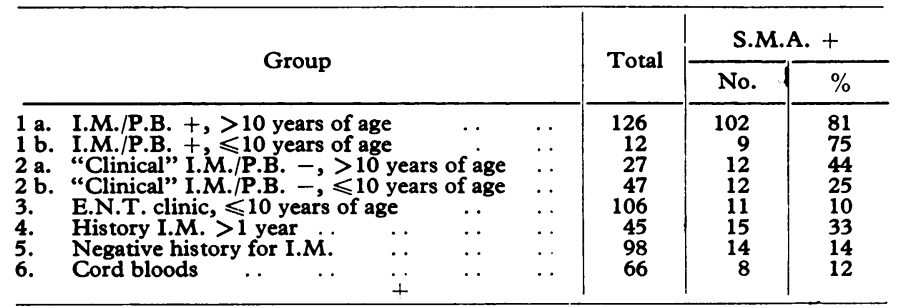

I.M./P.B. + = Infectious mononucleosis, Paul-Bunnell positive.

Follow-up of Group 1 a Patients.-Twenty-eight patients initially tested during the acute stage of their illness were retested after recovery at intervals which varied from 8 to 32 weeks. Of five negative for S.M.A. at the initial test four remained negative subsequently and one became positive after 20 weeks (immunoglobulin class not tested). Of the 23 positive at the initial test 13 were negative on retesting 4 to 32 weeks later and 10 were again positive when retested 8 to 38 weeks later. In six of these the immunoglobulin class of the antibody had changed from IgM to IgG, in one IgM antibody was still present, and in three the class was not determined.

E.B. Virus Antibody and S.M.A. in Control Children 10 Years Old or Less (Group 3).- The 106 sera in this group were screened for E.B. virus antibody. Forty-eight (45\%) gave a positive test for antibody to viral capsid antigen. The incidence of S.M.A. in the groups positive or negative for E.B. antibody was approximately equal $(5 / 48,6 / 58)$.

\section{Discussion}

This survey shows that in infectious mononucleosis S.M.A., predominantly of IgM class, are present in a large majority $(81 \%)$ of patients tested within one month of onset of symptoms. This is a similar incidence to that reported in viral hepatitis (Farrow et al., 1970). In the latter condition the sooner after onset sera are tested the higher the positivity rate for S.M.A. (Ajudkiewicz et al., 1972), and it may well be that this would hold for infectious mononucleosis as well.

After recovery S.M.A. may apparently persist in some subjects for a considerable time. The patients from whom follow-up sera could be obtained were a rather small group, but of the 23 initially positive for IgM S.M.A. 13 (56\%) were again positive when a second serum sample was tested 8 to 32 weeks later. In at least six of these the class of the antibody had changed to IgG. Funthermore, in a group of subjects with a history of Paul-Bunnell-positive infectious mononucleosis more than one year before testing (group 4) the incidence of S.M.A. was higher (33\%) than the $14 \%$ found in a group of similar age distribution (group 5) with negative histories. The figure for cond blood samples was similar (8/66 positives, or $12 \%$ ).

The question next arises why subjects with a negative history nevertheless show an appreciable incidence (14\%) of S.M.A.

It is known (Henle and Henle, 1967) that during adolescence and early adult life the incidence of antibody to E.B. virus rises steadily. Despite their negative history, therefore, the subjects in group 5 may have had undiagnosed clinical or unnoticed subclinical infectious mononucleosis which could have left a proportion of them with persistent S.M.A. To investigate this possibility we tested a group of children 10 years old or less for E.B. virus antibody and for S.M.A. The incidence of E.B. virus antibody in these children (group 3) was $45 \%$ and the incidence of S.M.A. $10 \%$, but no correlation between the two was evident. 
If, therefore, we take a negative test for E.B. virus antibody as indicating a truly negative history of infectious mononucleosis it appears that a clinical attack of infectious mononucleosis is only one of several possible causes of persisting serum S.M.A. Previous studies have indicated that some cases of viral hepatitis may also develop S.M.A. which persist in the serum for several months after recovery and that S.M.A. frequently accompany chronic autoimmune hepatitis and malignant disease. The findings in the children tested in the present study show that $(a)$ in Paul-Bunnellpositive infectious mononucleosis in childhood (group 1 b) the incidence of S.M.A. is approximately the same $(75 \%)$ as in adult cases; $(b)$ in "clinical" infectious mononucleosis in childhood with negative Paul-Bunnell tests (group 2 b) the incidence was somewhat raised $(25 \%)$ as compared with children with no clinical or serological evidence of infectious mononucleosis, where the incidence of S.M.A. was $10 \%$. It seems probable, therefore, that, at any rate in childhood, and perhaps also in adult life, there are other probably viral infections which may give rise to S.M.A. not only acutely but in some subjects persistently. It has been reported that cytomegalovirus infection may be one of these (Ajdukiewicz et al., 1972). The importance of extrinsic agents such as infection rather than genetic factors in determining the presence and lack of sex selectivity of S.M.A. was pointed out by Hooper et al. (1972), who drew attention to the contrast between S.M.A. and many other autoantibodies in these respects.

The role of hepatic damage in S.M.A. production requires further consideration in the light of the present findings. The presence of S.M.A. is linked in the literature with certain forms of liver disease, though Whitehouse and Holborow (1972) thought this association absent in S.M.A.-positive malignant disease. There is no doubt that liver cell damage occurs in infectious mononucleosis, whether clinically or merely biochemically manifest. It has been shown (Farrow et al., 1971) for several cell types that S.M.A.-positive sera may react specifically with a subcellular microfilamentous network associated with the cell surface, and it seems likely that this explains the polygonal pattern of liver staining seen with some of them. It has been shown, however, that absorption with crude actomyosin extracted from human myometrium effectively removes not only the antibodies in human sera which stain smooth muscle fibres but also the ability that some such sera have to produce polygonal and glomerular staining. It was suggested, therefore (Holborow, $1972 \mathrm{a}, \mathrm{b}$ ), that in certain viral infections antigenic modification of contractile proteins in the infected cells occurs which leads to production of autoantibodies recognizable by their specific reactivity with smooth muscle. In infectious mononucleosis such a process would presumably involve liver cells, but there is nothing liver-specific about cellular contractile proteins, and similar effects of virus in other cells-for example, the atypical lymphocytes-could equally contribute to production of polygonal and glomerular autoantibodies.

Our finding here that while most infectious mononucleosis sera give smooth muscle staining only some give polygonal or glomerular staining or both points to the likelihood that more than one antigenic specificity is involved in this postulated process of autoimmunization. Our experiments now in progress are investigating the staining patterns produced by antisera raised in rabbits against the various contractile protein subunits of smooth muscle; it is hoped to obtain information which will help identification of the different antigenic cellular components capable of giving rise to smonth muscle autoantibodies in human disease.

We are grateful to the general practitioners in Reading who helped with this investigation and to Dr. A. H. Tomlinson and Dr. M. S. Pereira for the E.B. virus antibody tests.

\section{References}

Ajdukiewicz, A. B., Dudley, F. J., Fox, R. A., Doniach, D., and Sherlock, S. (1972). Lancet, 1,803

Doniach, D., and Walker, J. G. (1969). Lancet, 1, 813.
Farrow, L. J., et al. (1970). British Medical fournal, 2, 693.

Farrow, L. J., Holborow, E. J., and Brighton, W. D. (1971). Nature New Biology, 232, 186.

Henle, G., and Henle, W. (1967). Cancer Research, 27, 2442.

Holborow, E. J., (1972 a). Proceedings of the Royal Society of Medicine,

65, 481.

Holborow, E. J. (1972 b). British Medical Bulletin, 28, 142 . D. H. (1972). Clinical and Experimental Immunology, 12, 79.

Johnson, G. D., Holborow, E. J., and Glynn, L. E. (1965). Lancet, 2, 878. W, 511 .
Whitehouse, J. M., and Holborow, E. J. (1971). British Medical fournal, 